\title{
CALIBRATING THE STIFFNESS OF AFM CANTILEVERS VIA INSTRUMENTED INDENTATION DEVICES
}

\author{
${ }^{1}$ Anna CHARVÁtOVÁ CAMPBELL, 1,2Marek HAVLÍČEK, 1,2Petr KLAPETEK \\ ${ }^{1}$ Czech Metrology Institute, Brno, Czech Republic, EU, acharvatovacampbell@cmi.cz \\ ${ }^{2}$ Central European Institute of Technology, Brno, Czech Republic, EU
}

https://doi.org/10.37904/nanocon.2021.4377

\begin{abstract}
In recent years the study of mechanical properties using atomic force microscopy (AFM) has become very popular. As it uses much lower forces than nanoindentation methods it allows to focus on smaller volumes which makes it an excellent tool for the study of thin films and the creation of high-resolution maps. Although it gives a quantitative as well as a qualitative analysis some open problems remain in the quantitative aspects. One of the problems encountered is related to the stiffness of the AFM cantilever. Most microscopes offer builtin methods for the calibration of cantilever stiffness. Unfortunately, these are often insufficient from the point of view of metrology. Uncertainties and traceability are rarely discussed.

Alternatively, the stiffness of a cantilever can be determined using a nanoindentation device. This well-known method offers simpler uncertainty analysis and traceability. In this contribution we explore the possibilities of this method from the metrological point of view. We shall present an uncertainty budget with focus on repeatability and we shall also discuss traceability issues.
\end{abstract}

Keywords: AFM, mechanical properties, calibration

\section{INTRODUCTION}

The study of mechanical properties using atomic force microscopy (AFM) has become very popular in recent years. While it has become a standard tool for the study of biological samples (e.g. [1-5]), stiffer samples are studied less frequently.

For a quantitative analysis the value of the cantilever spring constant must be known. However, this value is not trivial to achieve. The estimates given by probe manufacturers may vary up to hundreds of percent making them almost useless for quantitative analysis. Several methods for the calibration of the spring constant have been developed, for a review see e.g. [6,7]. AFM instruments usually have some method built-in, often Sader's method or the thermal vibration method. However, these methods work well only for soft cantilevers, as used in biological applications, and are not suitable for stiff cantilevers. Reference cantilevers can be used but are often not stiff enough for applications in material sciences. The traceability of these methods is rarely studied but is crucial for the quantitative analyses: without traceability the comparison of results between different instruments or laboratories is highly questionable.

One of the methods which seems most promising in terms of traceability is the method proposed by Clifford and Seah [8] which uses an indentation device to bend an AFM cantilever. Indentation devices are constructed for well-controlled application of load while recording precisely both the applied load and displacement. The traceability of the cantilever then follows from the traceability of the load and displacement sensors.

In this contribution we present a detailed case study of the determination of the spring constant of a stiff AFM cantilever. The uncertainty budget of all steps in the analyses is presented, as well as repeatability and traceability issues. The applicability of this method for a given cantilever depends also on the ranges of the 
indentation device. Indentations instead of bending might occur or the limits of the mathematical model this method is based on may be reached. Some suggestions how to estimate the impact of these effects are made.

\section{METHODS}

In this work we follow the method described in [8]. We shall briefly present its main idea. It is based on the well-known model of the small elastic deformations on an ideal rectangular homogenous beam with a fixed end (e.g. [9,10]). Also, the beam is considered massless [9]. In this case the deformation $\Delta z$ is proportional to the applied force $\mathrm{F}$ as

$\Delta \mathrm{z}=\frac{L^{3}}{3 E J_{z}} F$,

where $L$ is the length of the beam, $E$ its Young's modulus and $J_{z}$ the moment of inertia about the neutral axis (along the length). For a rectangular cross section this is $\mathrm{J}_{\mathrm{z}}=\mathrm{wt} \mathrm{t}^{3} / 12$.

Equation (1) holds for a general position of the load allowing to obtain the stiffness from multiple points by fitting the relation

$X=p\left(\frac{d F}{d z}\right)^{-\frac{1}{3}}+X_{0}$.

Here $X_{0}$ is the possible difference between the apparent fixed end and the true position. The stiffness can be obtained from this as

$\mathrm{k}=\frac{p^{3}}{\left(L-X_{0}\right)^{3}}$.

Solutions using for large deformations exist in literature as well, see e.g. $[11,12]$. Unfortunately, it is not possible to find a simple relation between deformation and load. Instead, numerical solutions involving inversions of elliptic integrals are used [13].

The procedure in [8] does not take into account the displacement into the cantilever due to indentation. The total compliance measured by the indentation device, i.e., the inverse of the derivative of the loading curve, is the sum of the compliance of the cantilever and the compliance of contact. Assuming the surface of the cantilever is homogenous along its length we can measure the compliance of contact on the fixed part of the cantilever.

Experiments were performed using the Ultra Hardness Nano Tester from Anton Paar. The instrument has been calibrated traceably as described in [14]. The resulting linear calibration constants for the force and depth sensor have a relative uncertainty of $12 \%$ and $1 \%$. The optical camera and the mechanical moving stage allow placements with an uncertainty approx. $0.5 \mathrm{um}$. Both were calibrated using a calibration grating.

One measurement of the cantilever's spring constant requires the determination of the coordinates of the points defined in Figure 1.

A reference indent I on the fixed part of the cantilever was made in order to align precisely the position of the indenter with respect to the optical camera. A set of loading curves was measured at different positions $X_{1}, \ldots, X_{N}$. Further indentations $I_{1}, \ldots, I_{M}$ were performed on the fixed part of the cantilever to assess the contact stiffness. In all cases a linear loading, linear unloading scheme was used. For bending we used very low maximum loads, usually 50 or $70 \mu \mathrm{N}$ which are close to the lower limit of our instrument. For indentations we used the same values in order to be able to use directly the contact stiffness. For the reference indent we used $2 \mathrm{mN}$, which was one of the lowest values for which the resulting indent could be reliably identified in the optical microscope. 


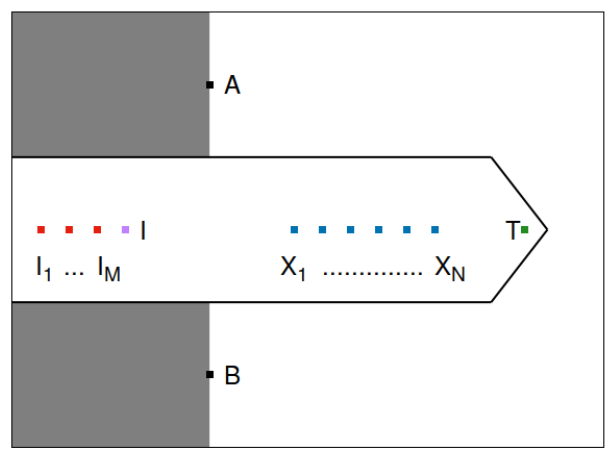

Figure 1 Plan of the measuring points on the cantilever. T - position of tip, A, B - positions on the edge, $\mathrm{X}_{1}, \ldots, \mathrm{X}_{\mathrm{N}}$ - position of bending measurements, $\mathrm{I}_{1}, \ldots, \mathrm{I}_{\mathrm{M}}$ - positions of indentation measurements.

Data processing was based on the opensource Niget [15] (force-distance curve processing) and the opensource algorithm OEFPIL, obtainable at https://cran.r-project.org/package=OEFPIL. The OEFPIL "Optimal Estimate for Function Parameters by Iterated Linearization" algorithm allows to take into account a general covariance matrix, in contrast to ordinary least squares minimization.

Different commercial cantilevers produced mainly by Bruker have been used for testing. The main case study in this work was performed on a stiff cantilever RTESPA525 from Bruker.

\section{RESULTS AND DISCUSSION}

The contact stiffness was found to be $(87.9 \pm 2.5) \mathrm{nm} / \mathrm{mN}$ at $50 \mu \mathrm{N}$. For the following quantitative analysis we chose to use the same loading force as used for the bending. This allows us to avoid the conversion to the Young's modulus which involves the tip shape and would lead to an unnecessary increase of the uncertainties. An example of the set of acquired force distance curves is shown in Figure 2. The corresponding slopes both corrected and uncorrected are plotted together with the distance from the edge in the same figure.
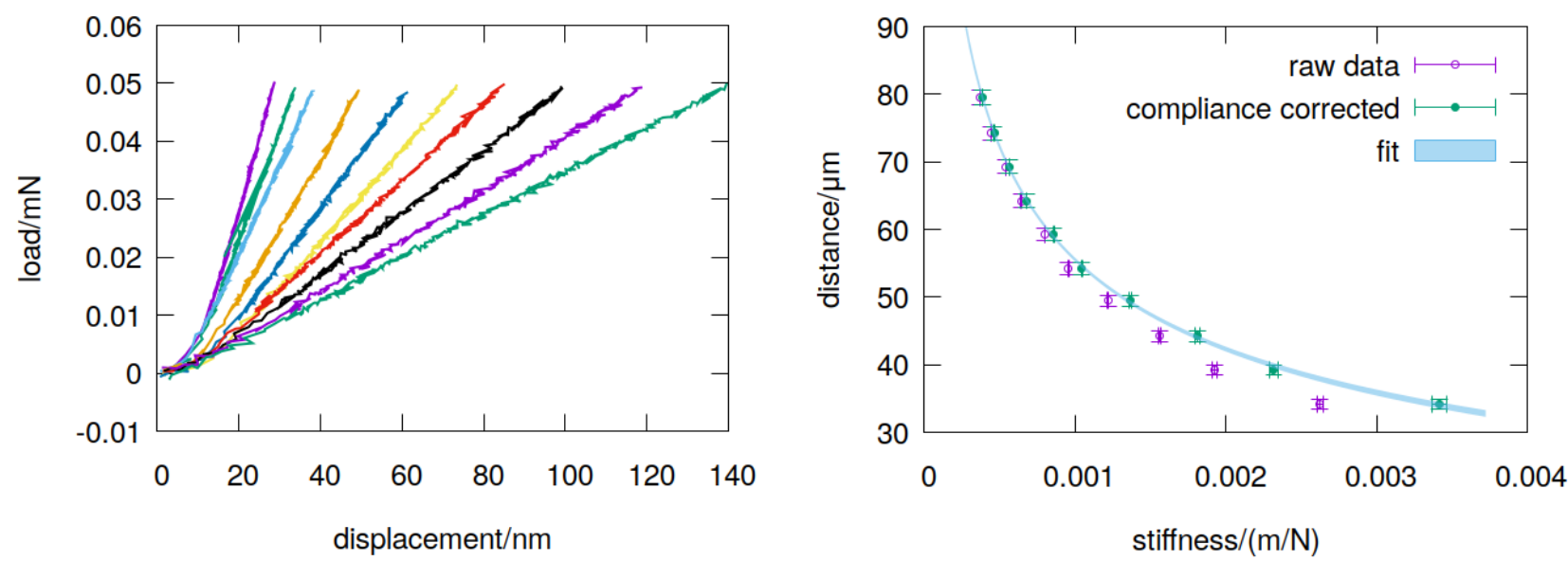

Figure 2 Example of unloading curves measured at different locations (left), corresponding plot of the distance versus the measured stiffness (right).

The resulting spring constant of the cantilever for this particular dataset is $(140 \pm 18) \mathrm{N} / \mathrm{m}$.

The apparent compliance measured at the point closest to the fixed edge was in this particular case $380 \mathrm{~nm} / \mathrm{mN}$, thus the contact compliance is more than $20 \%$ of the measured compliance. We can see that although the force-distance curves seem linear at first sight the correction for the contact stiffness has a 
noticeable impact. It shifts the cantilever spring constant from (146 \pm 18$) \mathrm{N} / \mathrm{m}$ without the correction to (140 \pm 18) $\mathrm{N} / \mathrm{m}$, thus ignoring the indentation introduces an overestimate of approx. $4 \%$.

The uncertainty of the slopes of the unloading curves was around $1.5 \%$, usually well below $3 \%$.

The cantilever length and the distance from the edge were calculated from the coordinates measured according to Figure 1. The corresponding uncertainties were calculated assuming an uncertainty in all coordinates equal to $0.5 \mu \mathrm{m}$, with the exception of the coordinates of the tip which are assumed to be $1.0 \mu \mathrm{m}$. This is necessary as the shape of the tip is not visible in the optical microscope. The average uncertainty of the length was $1.7 \mu \mathrm{m}$. For the total case study, the length was $(115.5 \pm 0.4) \mu \mathrm{m}$.

The spring constant of the cantilever is being calibrated with a factor $\mathrm{C}=\mathrm{C}_{\mathrm{F}} / \mathrm{Ch}_{\mathrm{h}}$, given by the calibration constants of the sensors. For our instrument it is $1.01 \pm 0.12$. This is due to a large uncertainty in the calibration of the force sensor which will be the focus of our future calibration studies.

For our case study the fitting parameters $\left(p, X_{0}\right)$ were found to have uncertainties approx. $2-3 \%$ for $p$ and around $15-20 \%$ for $\mathrm{X}_{0}$. They are strongly correlated, with a correlation around -0.98 .

The uncertainty of the spring constant is given by the combination of the uncertainties due to the uncertainties of the calibration factor, length and fitting parameters

$\mathrm{u}(\mathrm{k})=\sqrt{\left(\frac{\partial k}{\partial c}\right)^{2} u(c)^{2}+\left(\frac{\partial k}{\partial L}\right)^{2} u(L)^{2}+\left(\frac{\partial k}{\partial p}\right)^{2} u(p)^{2}+\left(\frac{\partial k}{\partial X_{0}}\right)^{2} u\left(X_{0}\right)^{2}+\left(\frac{\partial k}{\partial p}\right)\left(\frac{\partial k}{\partial X_{0}}\right) \operatorname{corr}\left(p, X_{0}\right) .}$

Here corr $\left(\mathrm{p}, \mathrm{X}_{0}\right)$ is the correlation between the two fitting parameters. In all cases we encountered it was negative thus lowering the total uncertainty.

The uncertainty budget for our example is shown in Table 1.

Table 1 Example uncertainty budget

\begin{tabular}{|c|c|c|c|c|}
\hline Variable & $\begin{array}{c}\text { Value of variable } \\
\mathbf{X}_{\mathbf{i}}\end{array}$ & $\begin{array}{c}\text { Value of standard } \\
\mathbf{u n c e r t a i n t y} \\
\mathbf{u}\left(\mathbf{x}_{\mathbf{i}}\right)\end{array}$ & $\begin{array}{c}\text { Sensitivity coefficient } \\
\frac{\boldsymbol{\partial} \boldsymbol{k}}{\boldsymbol{\partial} \boldsymbol{x}_{\boldsymbol{i}}}\end{array}$ & $\begin{array}{c}\text { Contribution to } \\
\text { uncertainty } \\
(\mathbf{N} / \mathbf{m})\end{array}$ \\
\hline Calibration factor c & 1.01 & 0.12 & $\frac{p^{3}}{\left(L-X_{0}\right)^{3}}$ & 16 \\
\hline $\begin{array}{c}\text { Length } \\
\mathrm{L}\end{array}$ & $115.3 \mu \mathrm{m}$ & $1.7 \mu \mathrm{m}$ & $-3 c \frac{p^{3}}{\left(L-X_{0}\right)^{4}}$ & 6 \\
\hline Fitting parameter $\mathrm{p}$ & $640 \mathrm{~N}^{1 / 3} \cdot \mathrm{m}^{2 / 3}$ & $13 \mathrm{~N}^{1 / 3} \cdot \mathrm{m}^{2 / 3}$ & $3 c \frac{p^{2}}{\left(L-X_{0}\right)^{3}}$ & \\
\hline $\begin{array}{c}\text { Fitting parameter } \\
\mathrm{X}_{0}\end{array}$ & $-8.6 \mu \mathrm{m}$ & $1.3 \mu \mathrm{m}$ & $3 c \frac{p^{3}}{\left(L-X_{0}\right)^{4}}$ & \\
\hline
\end{tabular}

We can see from Table 1 that the largest contribution to the uncertainty is due to the calibration factor. Its uncertainty is fairly high and future work will attempt to decrease it. The uncertainty due to the fitting parameters includes mainly the uncertainties due to sensor noise and positioning, the effect of the uncertainty of the contact stiffness is negligible. There is little room for improvement in positioning and sensor noise. The contributions due to length and fitting make up for a relative uncertainty of roughly $5 \%$. The total uncertainty is around $15 \%$ currently so in order to decrease our total spring constant uncertainty below $10 \%$ we need to achieve a calibration uncertainty approx. $8 \%$.

The case study included a number of experiments. We evaluated the repeatability on a set of measurements between which the instrument had been restarted or the cantilever moved. We found a standard deviation of $6 \mathrm{~N} / \mathrm{m}$ which corresponds to $4 \%$. This is significantly smaller than the now dominant uncertainty due to the 
instrument calibration but comparable with the uncertainty due to length. Based on our long-term experience with this instrument we assume that this reflects the small differences in the attachment of the cantilever as well as time instabilities in the calibration constants.

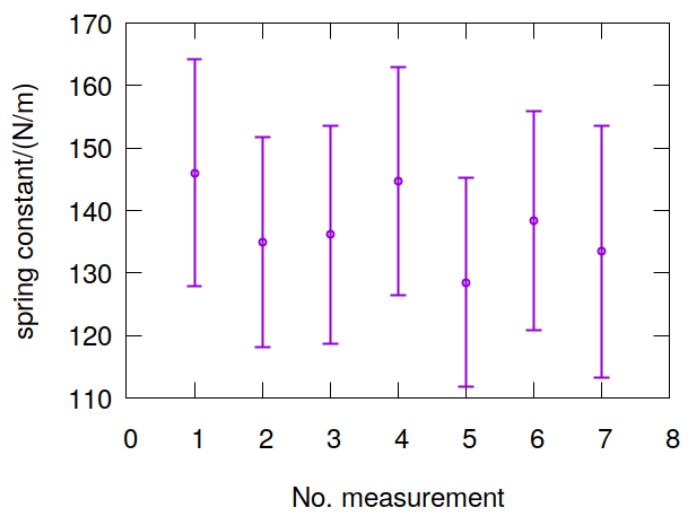

Figure 3 Repeatability of the measurement of the spring constant.

The number and location of the points where the bending was performed obviously influences the fit, and leads to differences in the fitting parameters and their covariance matrix. Obviously, it is important to choose the range of displacements as large as possible, as omitting the end points has a larger impact than omitting points in the middle. Numerical tests were performed by omitting combinations of points from the measured data set. However, even when using only 4 points for the fit the differences compared to the value obtained from the full data didn't exceed $7 \%$. The relative uncertainties ranged from $13 \%$, same as for the full data, to $18 \%$ in the worst case. Larger differences and uncertainties occurred when several neighboring points were omitted. This shows that an insufficient number of measuring points may introduce errors on the order of a few percent.

The limits of this model should be kept in mind. Notably, the assumption of small deformations should be checked. The large deformation model $[11,12]$ uses a dimensionless parameter $\alpha$

$\alpha=\sqrt{\frac{F L^{2}}{E J_{z}}}$

Comparing the model with the large deformation model $[11,12]$ it can be seen that in order to keep the deviation of the deformation below $1 \%$, a must be smaller than approx. 0.55 . Since the exact parameters, such as thickness and Young's modulus, are not known well, only very rough estimates can be made. We use nominal values for length, width and thickness and literature values for Young's modulus for the estimates. In order to keep a low, the lowest load at which the indentation instrument can operate should be used; for our instrument this is $50 \mu \mathrm{N}$. While for a typical stiff cantilever with nominal stiffness $200 \mathrm{~N} / \mathrm{m}$ we find for $\alpha$ the value 0.28 , the value is 2.04 for a typical soft cantilever. These estimates refer to the application of load at the end of the cantilever. If the load is applied closer to the fixed side the a value is reduced. Obviously for soft cantilevers care must be taken to choose appropriate positions to apply load. If the deformations become too large, the large deformation model would have to be used which requires significantly more complex data processing.

\section{CONCLUSION}

Although calibration of AFM cantilevers using indentation devices is a long-established method, details on the uncertainties and other aspects are rarely discussed in literature. General recommendations are difficult to give as they depend on the technical parameters of the instrument and the stiffness of the cantilever. In this contribution we illustrated some metrological aspects such as uncertainty analysis, corrections due to indentation and repeatability on a case study using a commercial cantilever. For our instrument the 
uncertainties in the calibration of the force and displacement sensors were the dominant contribution to the total uncertainty. It was also necessary to correct for the contact stiffness which could be measured on the fixed part of the cantilever. Repeatability has been checked as the attachment of the cantilever may vary and sensors can show time dependencies. However, differences were within margins of uncertainty. An uncertainty around $15-20 \%$ can be achieved by this method but this includes full traceability. The disadvantages are its demands for time and cantilever manipulation.

\section{ACKNOWLEDGEMENTS}

This work is supported by the Czech Science Foundation project GA19-15240S. " Multifunctional Nanocomposite Polymer Thin Films with Controlled Surface and Mechanical Properties Deposited in RF Dusty Plasma".

\section{REFERENCES}

[1] SANTOS, N.C., CASTANHO, M.A.R.B. An overview of the biophysical applications of atomic force microscopy. Biophysical Chemistry. 2004, vol. 107, pp. 133-149.

[2] PANG, D., THIERRY, A. R., DRITSCHILLO, A. DNA studies using atomic force microscopy: capabilities for measurement of short DNA fragments. Frontiers in Molecular Biosciences. 2015, vol. 2.

[3] HILAL, N., BOWEN, W.R., ALKHATIB, L., OGUNBIYI, O. A Review of Atomic Force Microscopy Applied to Cell Interactions with Membranes. Chemical Engineering Research and Design. 2006, vol. 84, pp. $282-292$.

[4] MAIN, K.H.S., PROVAN, J.I., HAYNES, P.J. et al. Atomic Force Microscopy - A tool for structural and translational DNA research. APL Bioengineering. 2021, vol. 5.

[5] HAASE, K., PELLING, A.E., Investigating Cell Mechanics With Atomic Force Microscopy. J. R. Soc.Interface. 2014, vol. 12.

[6] BURnhAM, N. A., CHEN, X., H ODGES, C. S., MATEI, G. A. THORESON, E. J., ROBERTS, C. J., DAVIES. M. C., TENDLER, S. J. B. Comparison of calibration methods for atomic-force microscopy cantilevers. Nanotechnology. 2003, vol. 14, pp. 1-6.

[7] GIBSON, C.T., WATSON, G.S., MYHRA, S. Scanning force microscopy - calibrative procedures for best practice. Scanning. 2006, vol 19, pp. 564 - 581.

[8] CLIFFORD, C.A., SEAH, M.P. Improved methods and uncertainty analysis in the calibration of the spring constant of an atomic force microscope cantilever using static experimental methods. Measurement Science Technology. 2009, vol. 20, p. 125501.

[9] FEYNMAN, R., LEIGHTON, R., SANDS, M., The Feynman lectures on Physics: mainly Electromagnetism and Matter. Reading: Addison-Wesley, 1989.

[10] TIMOSHENKO, S.P. History of the Strength of Materials. New York: Dover, 1983.

[11] BISSHOPP, K. E., DRUCKER, D. C. Large deflections of cantilever beams. Q. Appl. Math. 1945, vol. 3, pp. 272275.

[12] BELENDEZ, T., NEIPP, C., BELENDEZ, A. Large and small deflections of a cantilever beam. European Journal of Physics. 2002, vol. 23, pp. 371-379.

[13] CHEN, L. An Integral Approach for Large Deflection Cantilever Beams. International Journal of Non-Linear Mechanics. 2010, vol.45, pp. 301 - 305.

[14] CAMPBELLOVÁ, A., VALTR, M., ZU゚DA, J. and KLAPETEK, P. Traceable measurements of small forces and local mechanical properties. Meas. Sci. Technol. 2011, vol. 22, pp. 094007-094013.

[15] CHARVÁtOVÁ CAMPBELL, A., GROLICH P., ŠLESINGER, R. Niget: Nanoindentation general evaluation tool. Software X. 2019, vol. 9, pp. 248-254. 